\title{
Mechanical Circulatory Support Devices for Cardiogenic Shock: State of the Art
}

\author{
Ludhmila Abrahao Hajjar ${ }^{1 *}$ and Jean-Louis Teboul ${ }^{2}$
}

\begin{abstract}
This article is one of ten reviews selected from the Annual Update in Intensive Care and Emergency Medicine 2019. Other selected articles can be found online at https://www.biomedcentral.com/collections/ annualupdate2019. Further information about the Annual Update in Intensive Care and Emergency Medicine is available from http://www.springer.com/ series/8901.
\end{abstract}

\section{Introduction}

Cardiogenic shock is the clinical expression of circulatory failure, as a consequence of left, right or biventricular dysfunction. Cardiogenic shock is also defined as a state of critical end-organ hypoperfusion due to primary cardiac dysfunction [1-3]. Cardiogenic shock is not simply a decrease in cardiac contractile function, but also a multiorgan dysfunction syndrome involving the entire circulatory system, often complicated by a systemic inflammatory response syndrome with severe cellular and metabolic abnormalities [4]. The clinical presentation of cardiogenic shock varies from hemodynamic abnormalities of pre-shock to mild shock, progressing to more profound shock and finally refractory shock, which is associated with high mortality rates. Additional insults can occur, such as arrhythmias, vasodilation, ischemia and infection, acutely changing the trajectory of the disease [5]. The contemporary management of cardiogenic shock involves early diagnosis and directed therapy to optimize oxygen delivery and tissue perfusion.

The diagnosis of cardiogenic shock is based on the presence of: (1) persistent hypotension defined as systolic blood pressure $<90 \mathrm{mmHg}$ or mean arterial pressure (MAP) $30 \mathrm{mmHg}$ below baseline or requirement of vasopressors to achieve a systolic blood pressure $\geq$

\footnotetext{
* Correspondence: ludhmila@usp.br

1 Department of Cardiopneumology, Instituto do Coracao, Universidade de

São Paulo, Hospital SirioLibanes, São Paulo, Brazil

Full list of author information is available at the end of the article
}

$90 \mathrm{mmHg}$; and (2) signs of impaired organ perfusion (e.g., central nervous system abnormalities including confusion or lack of alertness, or even loss of consciousness; oliguria; cold, clammy skin and extremities, tachypnea, increased arterial lactate $>2 \mathrm{mmol} / \mathrm{L}$ ) despite normovolemia or hypervolemia [1].

Some clinical trials include hemodynamic parameters as diagnostic criteria, including low cardiac index (CI) $\left(<1.8 \mathrm{~L} / \mathrm{min} / \mathrm{m}^{2}\right.$ without support or $<2.2 \mathrm{~L} / \mathrm{min} / \mathrm{m}^{2}$ with support) and adequate or elevated filling pressures (left ventricular end-diastolic pressure $>15 \mathrm{mmHg}$ ) $[1,6]$. However, the diagnosis of cardiogenic shock is mainly based on clinical examination.

The most common cause of cardiogenic shock is acute coronary syndrome. Other causes include mechanical complications of acute coronary syndrome, myocarditis, right ventricular (RV) failure and progressive heart failure from cardiomyopathies [7]. In the CardShock study [8], among 219 patients with cardiogenic shock, 68\% had ST-elevation myocardial infarction (STEMI), 9\% mechanical complications of myocardial infarction and 20\% non-acute coronary syndrome causes, such as worsening chronic heart failure, valvular heart disease, stress-induced cardiomyopathy and myocarditis. Patients may also present with cardiogenic shock post-cardiotomy and because of significant ventricular arrhythmias, pulmonary embolism, or cardiac tamponade.

Cardiogenic shock remains a challenging condition with mortality rates of approximately 50\% [4]. The field of temporary mechanical circulatory support to manage patients with cardiogenic shock has advanced in the last decade. However, indications for temporary mechanical circulatory support and device selection are part of a complex process requiring consideration of the severity of cardiogenic shock, early and prompt hemodynamic resuscitation, specific patient risk factors, technical limitations, adequate resources and training, and assessment of futility of care. Early intervention with adequate selection of the most appropriate mechanical circulatory support device may improve outcomes [5] (Fig. 1). 


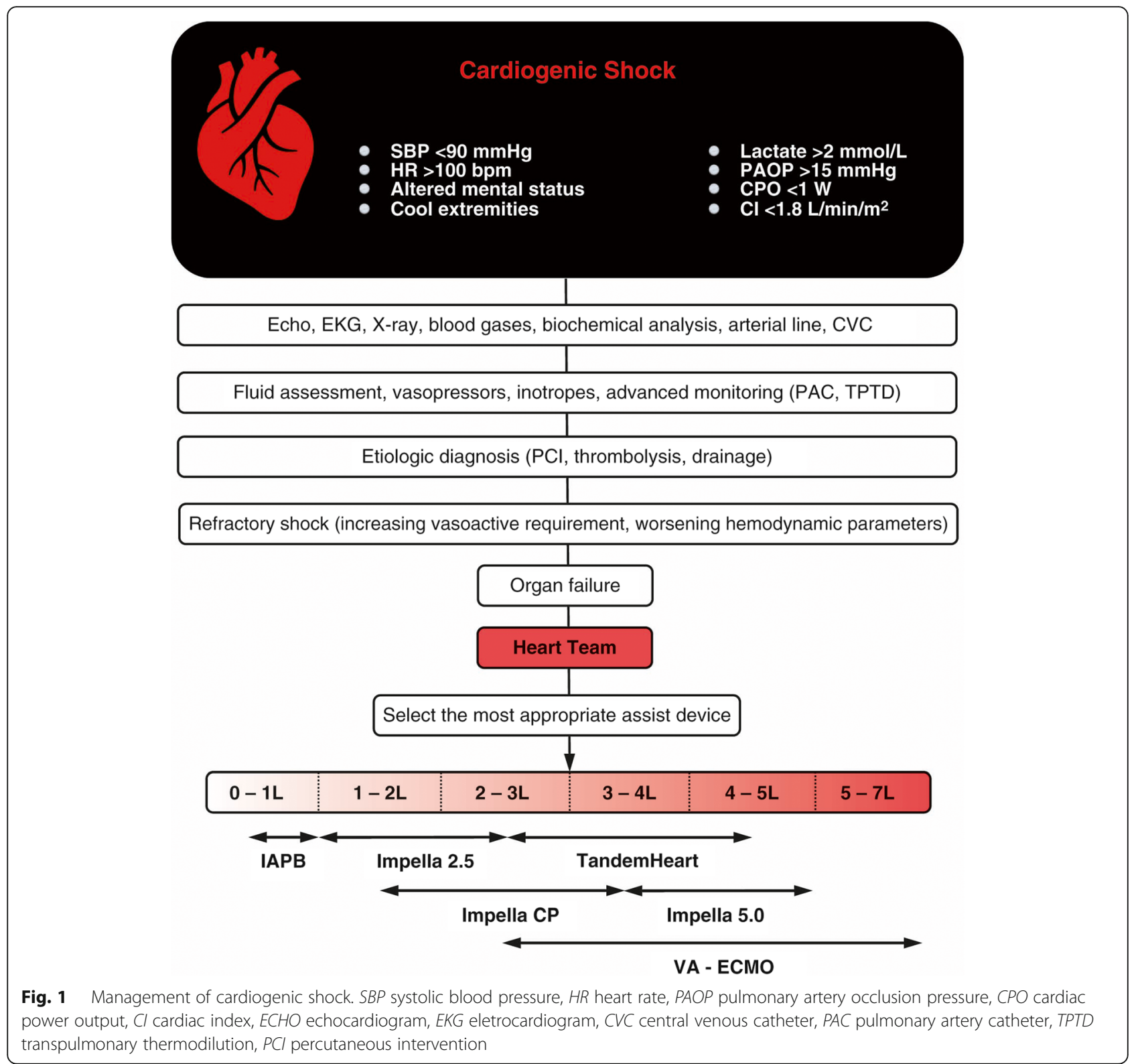

\section{Management of Cardiogenic Shock}

The focus of the treatment of cardiogenic shock is to prevent organ failure through hemodynamic resuscitation and optimization, and simultaneously to assess and treat a potential reversible cause.

\section{Hemodynamic support}

Myocardial dysfunction generates a spiral of reduced stroke volume, increasing ventricular diastolic pressure and wall stress, which reduces coronary perfusion pressure. In addition, left ventricular (LV) dysfunction and ischemia increase diastolic stiffness, which elevates left atrial pressure, leading to pulmonary congestion, hypoxia, and worsening ischemia. Initial compensatory mechanisms result in activation of the sympathetic nervous system and the renin-angiotensin-aldosterone system. In about $40-50 \%$ of cases, there is a concomitant activation of cytokines, increased inducible nitric oxide synthase (iNOS), increasing levels of $\mathrm{NO}$ and subsequent vasodilation and hypotension. Activation of the inflammatory cascade is associated with myocardial depression. Cytokines such as interleukin (IL)-1, IL-6 and tumor necrosis factor (TNF)- $\alpha$ and reactive oxygen species (ROS) can disturb regional autoregulation leading to impaired microcirculation. This might also contribute to reduced oxygen extraction at the tissue level [7]. Improvement in hemodynamic parameters, which reflects normalization of the macrocirculation, does not always result in restoration of the microcirculation [9]. This has been shown by studies in conditions of a loss 
of hemodynamic coherence where resuscitation resulted in normalization of systemic hemodynamic variables but did not lead to an improvement in microcirculatory perfusion and oxygenation [10,11].

We can divide the hemodynamic management of patients with cardiogenic shock into three steps.

\section{Step 1: Initial assessment (salvage phase)}

Recognition of cardiogenic shock should trigger a rapid assessment of the etiology and hemodynamic profile based on history, physical examination, laboratory investigations, electrocardiogram (EKG), and echocardiography. The initial assessment and management are performed wherever the patient is-emergency room, hemodynamic lab, surgical room or intensive care unit (ICU). Even in cases of prehospital diagnosis, adequate management should be started in this salvage phase.

Arterial line: arterial blood pressure must be monitored using a continuously transduced arterial line. The initial target MAP should be at least $65 \mathrm{mmHg}$, taking into account individual variability. Radial or femoral access is preferred in this setting. An arterial line also allows blood gas analysis and arterial lactate monitoring. The arterial catheter provides calculation of pulse pressure variation (PPV), which is a predictor of fluid responsiveness in mechanically ventilated patients when applicable. Minimally invasive cardiac output might be useful in the initial management of patients, using devices based on arterial waveform analysis. However, a number of studies have shown inconsistent performance of these devices in the setting of acute/very low cardiac output states and cardiogenic shock [12].

Central venous catheter: the central venous catheter (CVC) allows fluids, inotropes and vasopressors to be administered in a timely fashion. From the CVC, important hemodynamic variables, such as the central venous pressure (CVP), the central venous oxygen saturation $\left(\mathrm{ScvO}_{2}\right)$ and the central venous carbon dioxide pressure $\left(\mathrm{PcvCO}_{2}\right)$ can be obtained. The CVC must be inserted preferentially in the internal jugular vein, using ultrasound guidance.

Echocardiography: in the context of cardiogenic shock, transthoracic echocardiography is essential for diagnosis and to follow therapy. In the ICU, repeated echocardiography is recommended for evaluation of left and right ventricular function, valve dysfunction, and exclusion/ diagnosis of mechanical complications. Echocardiography is also emerging as a hemodynamic monitoring tool in the ICU for estimation of cardiac output, cardiac filling pressures, to predict volume responsiveness, and determine response to critical care interventions [13]. The main advantages of echocardiography are its non-invasiveness and its ability to assess both cardiac structure and function. From measurement of the velocity-time integral (VTI) of the flow in the LV outflow tract, measurement of RV size, search for pericardial effusion and search for respiratory variations in vena cava diameter, intensivists can quickly confirm and/or define the type of shock. Moreover, changes in cardiac output induced by therapeutic tests of fluid responsiveness or by fluid administration can be reliably estimated by the changes in VTI, as the area of the LV outflow tract remains unchanged over a short time period [12].

Vasopressors: norepinephrine is considered the vasopressor of choice in cardiogenic shock. Subgroup analysis of patients with cardiogenic shock suggested lower mortality with norepinephrine compared to dopamine [14]. It is speculated that the increased heart rate associated with dopamine use may contribute to increased ischemic events. In a recent study of patients with septic shock, norepinephrine administration during early resuscitation increased cardiac systolic function despite the presumed increase in LV afterload secondary to the increased arterial pressure [15]. In patients with vasodilatory shock refractory to norepinephrine (doses $>0.2 \mu \mathrm{g} / \mathrm{kg} / \mathrm{min}$ ), vasopressin should be initiated. This drug is associated with reduced rates of atrial fibrillation and lower requirement of renal replacement therapy (RRT) in vasodilatory shock [16]. Epinephrine should not be used in cardiogenic shock due to its effect in increasing lactate levels, in increasing oxygen consumption and arrythmias and also due to the association with higher mortality rates $[17,18]$.

In a recent randomized clinical trial, norepinephrine was superior to epinephrine in achieving hemodynamic goals, and epinephrine increased lactate levels in patients with cardiogenic shock after acute myocardial infarction [19]. A meta-analysis of individual data from 2584 patients showed that epinephrine use for hemodynamic management of patients with cardiogenic shock was associated with a threefold increased risk of death [20].

Inotropes: The addition of an inotropic agent may help to improve stroke volume after hemodynamic stabilization with a vasopressor in cardiogenic shock. Dobutamine is the initial therapy and (starting dose $2.5 \mu \mathrm{g} / \mathrm{kg} / \mathrm{min}$ ) may act rapidly to restore stroke volume [1]. Levosimendan and milrinone, due to their vasodilator properties, should not be used in the management of cardiogenic shock.

Fluids and red blood cells: due to the vasodilation that can occur in cardiogenic shock, many patients may develop hypovolemia. In these cases, dynamic assessment of fluid status should be performed, through echocardiography parameters and cardiac output monitors. A fluid challenge identifies and simultaneously treats volume depletion, whilst avoiding deleterious consequences of fluid overload through its small volume and targeted administration [21]. 
Red blood cell transfusion is recommended on an individual basis, according to hemodynamic parameters after the administration of inotropes and vasopressors and fluid status assessment. Usually, hemoglobin levels $>8 \mathrm{~g} / \mathrm{dL}$ are needed to optimize oxygen delivery in patients with shock. However, the value is dependent on hemodynamic circumstances, patient age, comorbidities and compensatory response to anemia and shock $[22,23]$.

\section{Step 2: Optimization and stabilization}

The second phase of the hemodynamic management of cardiogenic shock aims to optimize the hemodynamic goals and stabilize the patient to prevent complications. In this setting, accurate hemodynamic monitoring is recommended, and re-evaluation of therapies and patient response should be performed. The objectives of care are to optimize fluid status, and to adjust doses of inotropes and vasopressors to improve oxygen delivery and tissue perfusion.

Hemodynamic monitoring: after collecting information from clinical examination, CVP, arterial blood pressure, heart rate, gas analysis, echocardiography and minimally invasive cardiac output assessment, in most cases it is possible to make valid therapeutic decisions and select the most appropriate hemodynamic therapy. If the response of the patient is positive and shock is resolving, there is no need to add any further monitoring device. If the response is inadequate, it is recommended that more information be obtained using an advanced hemodynamic monitoring technique. It is also recommended that advanced hemodynamic monitoring should be used earlier when acute respiratory distress syndrome (ARDS) is associated with shock because in this situation fluid management is even more challenging. The two hemodynamic monitoring technologies considered as advanced are the pulmonary artery catheter (PAC) and transpulmonary thermodilution systems [12, 24]. PAC use has fallen out of favor over the last two decades because of the difficulty measuring and interpreting the hemodynamic variables as well as failure to demonstrate any benefit of its use in critically ill patients in randomized clinical trials [25]. Nevertheless, it has been recently suggested that the PAC may still have a key role in the hemodynamic monitoring of critically ill patients $[26$, 27]. Currently, the PAC is recommended in patients with refractory shock, in shock patients with RV dysfunction and/or with ARDS, and when selecting the ideal mechanical circulatory support device. Its advantage is that it can be used to measure the pulmonary artery pressure, and to provide an estimation of pulmonary vascular resistance and other potentially useful hemodynamic variables, such as right atrial pressure, left and right ventricular work, pulmonary artery occlusion pressure (PAOP), mixed venous oxygen saturation $\left(\mathrm{SvO}_{2}\right)$, oxygen delivery, oxygen consumption and extraction [12].
The use of transpulmonary thermodilution is recommended in patients with severe shock, especially in the case of ARDS [12, 24]. This technique measures cardiac output in an intermittent way, but transpulmonary thermodilution devices can also provide a real-time measurement of cardiac output through pressure waveform analysis after initial calibration. Pressure waveform analysis also continuously provides PPV and/or stroke volume variation (SVV), two dynamic markers of preload responsiveness. The cardiac output measurement is accurate and precise, even in patients with high blood flow RRT. The mathematical analysis of the thermodilution curve provides other hemodynamic variables, such as the global end-diastolic volume (GEDV), a marker of cardiac preload, the cardiac function index and the global ejection fraction, markers of cardiac systolic function. The extravascular lung water (EVLW) is a quantitative measure of pulmonary edema and the pulmonary vascular permeability index (PVPI), a marker of the lung capillary leak. Thus, such devices are particularly appropriate for guiding fluid management in patients with concomitant acute circulatory and respiratory failure as they help clinicians assess the benefit/risk ratio of fluid administration. The benefit can be evaluated by the preload responsiveness indices that these devices provide (PPV, SVV, pulse wave analysis-derived cardiac output response to passive leg raising or end-expiratory occlusion test). The recommendation to use advanced hemodynamic monitoring should apply only to the subgroup of patients with shock who do not respond to the initial treatment and/ or who have associated ARDS [12, 24].

\section{Step 3: Titrating therapies}

In this phase, patients should be carefully evaluated regarding the response to therapy. Clinical examination, macrohemodynamic and microhemodynamic variables, perfusion tissue markers and, if possible, microcirculation should be assessed in an integrative approach, trying to discern the circulatory status of patient. In the case of hemodynamic stability, vasoactive drug weaning may be started and de-escalating therapies might be a goal. However, $10-15 \%$ of patients develop refractory shock needing increased doses of vasopressors and inotropes, presenting signs of tissue hypoxia, and in many cases already developing organ failure. In this setting, mechanical circulatory support should be considered.

\section{Specific management according to the etiology of the cardiogenic shock}

In the case of cardiogenic shock due to acute coronary syndrome, myocardial revascularization is the only evidence-based therapy with proven survival benefit. Fibrinolysis should be reserved for patients with STEMI 
when timely percutaneous coronary intervention (PCI) is not feasible [2]. The SHOCK trial is one of the milestone randomized trials in cardiogenic shock [28]. Although it failed to show a reduction in 30-day mortality by early revascularization-based management either with PCI or coronary artery bypass grafting (CABG) [28], there was a significant mortality reduction at 6 months and at long-term follow-up [29]. More than $80 \%$ of patients with cardiogenic shock have multivessel or left main coronary artery disease [1]. These patients have higher mortality than patients with single-vessel disease. In recent years, immediate multivessel PCI of all high-grade lesions has been recommended in addition to the culprit lesion [2]. However, the randomized, multicenter Culprit Lesion Only PCI vs. Multivessel PCI in Cardiogenic Shock (CULPRIT-SHOCK) trial showed a significant clinical benefit of a culprit-lesion-only strategy with a reduction in the primary endpoint of 30-day mortality or severe renal failure requiring RRT (45.9\% culprit-lesion-only $\mathrm{PCI}$ vs. $55.4 \%$ immediate multivessel PCI group; relative risk 0.83; 95\% confidence interval $0.71-0.96 ; p=0.01$ ) which was mainly due to an absolute $8.2 \%$ reduction in 30-day mortality ( $43.3 \%$ vs. $51.5 \%$; relative risk 0.84 ; $95 \%$ confidence interval $0.72-0.98, p=0.03$ ) [30]. Two recent meta-analyses confirmed an increased mortality at short-term follow-up with multivessel PCI and similar outcome at longer follow-up [31]. Data from one-year outcomes from the CULPRIT-SHOCK trial showed that mortality did not differ significantly between the two groups at 1 year; however, the rates of rehospitalization for heart failure and repeat revascularization were higher in the culprit-lesio$\mathrm{n}$-only PCI group than in the multivessel PCI group at this time point [32].

Evidence comparing PCI vs. CABG showed that the type of revascularization did not influence the outcome of patients with cardiogenic shock [1]. In current clinical practice, immediate CABG is performed in less than $4 \%$ of patients [1]. Antithrombotic therapy, including antiplatelets and anticoagulation, is essential during and after PCI in patients with acute coronary syndrome. Prasugrel/ticagrelor or clopidogrel is indicated in addition to aspirin in all patients undergoing PCI. Because of the delayed and impaired onset of oral antiplatelets action in unstable patients, glycoprotein IIb/IIIa inhibitors or cangrelor may be more liberally used in cardiogenic shock [1].

Unfractionated heparin or low molecular weight heparin should also be administered with antiplatelets. There are no specific randomized trials in patients with cardiogenic shock, so the same recommendation applies as for other types of acute coronary syndrome [2]. The etiologic diagnosis is essential to improve outcomes. Echocardiography should be performed in the initial assessment of patients with cardiogenic shock to promptly diagnose and treat mechanical complications of acute myocardial infarction, cardiac tamponade, acute valve insufficiency or pulmonary embolism. Independent of the phase of therapy, organ support and maintenance must be given according to shock severity.

\section{Mechanical circulatory support}

Escalating doses of vasopressors and inotropes are associated with increased mortality. Mechanical circulatory support is an essential part of the management of cardiogenic shock and is commonly utilized as a bridge-to-decision, whether it is recovery, palliation, heart transplant or a durable mechanical circulatory support device. A comparison of technical features of the available percutaneous assist devices is shown in Table 1 .

\section{Intra-aortic balloon pump}

The intra-aortic balloon (IABP) pump, a counterpulsation pump placed percutaneously in the descending aorta, is the most used assist device worldwide. It requires a native beat and a stable rhythm as the balloon is synchronized with the heart. For more than four decades, IABP has been used to improve hemodynamic parameters in patients with cardiogenic shock. An IABP reduces afterload, increases cardiac output, optimizes coronary flow and decreases oxygen consumption [33]. The insertion of an IABP is relatively easy, it has a low cost and associated vascular complications are rare. However, the increase in cardiac output is relatively small, about $500-800 \mathrm{~mL} / \mathrm{min} / \mathrm{m}^{2}$. The IABPSHOCK II trial randomized 600 patients with cardiogenic shock after acute myocardial infarction and early revascularization to IABP or conventional treatment and found no difference in 30-day mortality between the treatment groups [34]. These results led to a downgrading of the IABP recommendation in the ESC guidelines with a current class IIIB recommendation for routine use of IABPs in cardiogenic shock. The 2017 ESC STEMI guidelines now recommend IABP use only in patients with mechanical complications (class IIa, level C) [35].

Recent meta-analyses of IABP therapy in post-acute myocardial infarction patients with cardiogenic shock (incorporating the results of the IABP-SHOCK II trial) have further called into question the utility of IABP therapy in these patients. Analyzing data from 17 studies, Romeo et al. reported no overall differences in short- or long-term mortality in patients receiving IABP therapy. Interestingly, when stratified by initial treatment, IABP therapy significantly reduced mortality (RR $0.77,95 \%$ CI $0.68-0.87$ ) in patients receiving thrombolytic therapy but significant increased mortality (RR 1.18, 95\% CI 1.04-1.34) in patients receiving primary PCI [36].

In spite of the evidence against the routine use of IABP, it is often used as initial mechanical support (bridge therapy) 
Table 1 Technical properties of percutaneous circulatory assist devices

\begin{tabular}{|c|c|c|c|c|c|c|}
\hline & IAPB & IMPELLA 2.5 & IMPELLA CP & IMPELLA 5.0 & TandemHeart & VA-ECMO \\
\hline Mechanism & Aorta & $\mathrm{LV} \rightarrow$ aorta & $\mathrm{LV} \rightarrow$ aorta & $\mathrm{LV} \rightarrow$ aorta & $\mathrm{LA} \rightarrow$ aorta & $\mathrm{RA} \rightarrow$ aorta \\
\hline Flow (L/min) & $0.3-0.5$ & $1.0-2.5$ & $3.7-4.0$ & Max. 5.0 & $2.5-5.0$ & $3.0-7.0$ \\
\hline Cannula size (Fr) & $7-8$ & $13-14$ & $13-14$ & 21 & $\begin{array}{l}15-17 \text { arterial } \\
21 \text { venous }\end{array}$ & $\begin{array}{l}14-16 \text { arterial } \\
18-21 \text { venous }\end{array}$ \\
\hline Femoral artery size $(\mathrm{mm})$ & $>4.0$ & $5.0-5.5$ & $5.0-5.5$ & 8.0 & 8.0 & 8.0 \\
\hline $\begin{array}{l}\text { Cardiac synchrony or } \\
\text { stable rhythm }\end{array}$ & Yes & No & No & No & No & No \\
\hline Maximum implant days & TBD & $7-10$ days & $7-10$ days & 2-3 weeks & 2-3 weeks & 3-4 weeks \\
\hline Cardiac power & $\uparrow$ & $\uparrow \uparrow$ & $\uparrow \uparrow$ & $\uparrow \uparrow$ & $\uparrow \uparrow$ & $\uparrow \uparrow \uparrow$ \\
\hline Afterload & $\downarrow$ & $\downarrow$ & $\downarrow$ & $\downarrow$ & $\uparrow$ & $\uparrow \uparrow \uparrow$ \\
\hline MAP & $\uparrow$ & $\uparrow \uparrow$ & $\uparrow \uparrow$ & $\uparrow \uparrow$ & $\uparrow \uparrow$ & $\uparrow \uparrow$ \\
\hline LVEDP & $\downarrow$ & $\downarrow \downarrow$ & $\downarrow \downarrow$ & $\downarrow \downarrow$ & $\downarrow \downarrow$ & $\leftrightarrow$ \\
\hline PAOP & $\downarrow$ & $\downarrow \downarrow$ & $\downarrow \downarrow$ & $\downarrow \downarrow$ & $\downarrow \downarrow$ & $\leftrightarrow$ \\
\hline LV preload & - & $\downarrow \downarrow$ & $\downarrow \downarrow$ & $\downarrow \downarrow$ & $\downarrow \downarrow$ & $\downarrow$ \\
\hline Coronary perfusion & $\uparrow$ & $\uparrow$ & $\uparrow$ & $\uparrow$ & - & - \\
\hline
\end{tabular}

IABP intraaortic balloon pump, VA-ECMO veno-arterial extracorporeal membrane oxygenation, $L V$ left ventricle, $L A$ left atrium, RA right atrium, MAP mean arterial pressure, $L V E D P$ left ventricular end-diastolic pressure, $P A O P$ pulmonary artery occlusion pressure

until other more sophisticated devices become available. Another field of interest is the use of IABP in patients receiving extracorporeal membrane oxygenation (ECMO) due to the afterload reduction. There are data showing benefits of IABP in patients with ECMO, with a decrease in PAOP, less pulmonary edema and increased survival [37].

\section{TandemHeart}

The TandemHeart (TandemLife, Pittsburgh, PA, USA) is a percutaneous centrifugal pump that provides mechanical circulatory support of up to $4 \mathrm{~L} / \mathrm{min}$ via a continuous flow centrifugal pump. Oxygenated blood is withdrawn from the left atrium via a $21 \mathrm{Fr}$ inflow cannula placed via trans-septal puncture and then re-injected into the lower abdominal aorta or iliac arteries via a 15-17 Fr outflow cannula [38]. The TandemHeart is inserted through the femoral vein and is advanced across the interatrial septum into the left atrium. The need for transseptal puncture is a potential limitation to its widespread use. In 2005, Thiele et al. reported their experience with TandemHeart therapy in patients with cardiogenic shock post-acute myocardial infarction [39]. Patients were randomized to hemodynamic support with either an IABP or the TandemHeart. The primary endpoint was hemodynamic improvement. While greater improvements in cardiac power and in cardiac index were seen in patients receiving the TandemHeart, 30 -day mortality was similar ( $43 \%$ vs. $45 \%, p=0.86$ ) in the two groups. In 2011, Kar et al. reported outcomes following TandemHeart insertion in 80 patients with cardiogenic shock post-acute myocardial infarction [40]. Almost half of these patients had undergone cardiopulmonary resuscitation (CPR) immediately before or at the time of implantation. TandemHeart insertion was associated with significant improvements in hemodynamic indices. The 30-day and 6-month mortality rates were 40.2 and $45.3 \%$, respectively. One patient died following wire-mediated perforation of the left atrium. Other complications included the need for blood transfusion (71\%), sepsis/systemic inflammatory response syndrome [SIRS] (29.9\%), bleeding around the cannula (29.1\%), gastrointestinal bleeding $(19.7 \%)$, coagulopathy $(11 \%)$, stroke $(6.8 \%)$, and device-related limb ischemia (3.4\%).

\section{Impella}

The Impella (AbioMed, Danvers, MA, USA) is a continuous, non-pulsatile, axial flow Archimedes-screw pump that provides active support by expelling aspirated blood from the left ventricle into the ascending aorta. Unlike an IABP, the Impella does not require EKG or arterial waveform triggering, facilitating stability even in the setting of tachyarrhythmias or electromechanical dissociation. Reported complications include device migration, device malfunction due to thrombosis, hemolysis, bleeding requiring transfusion, arrhythmias, limb ischemia, tamponade, aortic or mitral valve injury, and stroke $[1,38]$.

Three versions of Impella for LV support are available: the Impella LP 2.5 that can deliver $2.5 \mathrm{l} / \mathrm{min}$ of cardiac output, the Impella $\mathrm{CP}$ that can deliver $3.7 \mathrm{~L} / \mathrm{min}$ of cardiac output, and the Impella LP 5.0 that can deliver 5.0 l/ min of cardiac output. While the Impella LP 2.5 and Impella $\mathrm{CP}$ can be inserted percutaneously via a $12-14 \mathrm{Fr}$ sheath, insertion of the Impella LP 5.0 requires surgical 
cutdown of the femoral or axillary artery prior to insertion of a 22 Fr sheath [38].

The ISAR-SHOCK trial was a 2-center, randomized controlled pilot study that randomized 26 patients with cardiogenic shock post-acute myocardial infarction to Impella LP 2.5 or IABP therapy [41]. Although the cardiac index after $30 \mathrm{~min}$ of support was significantly increased in patients with the Impella LP 2.5 compared to those with an IABP $\left(0.49 \pm 0.46\right.$ vs. $0.11 \pm 0.31 \mathrm{~L} / \mathrm{min} / \mathrm{m}^{2}$ respectively; $p=0.02$ ), at $4 \mathrm{~h}$, no significant differences were seen in the cardiac index, modified cardiac power index (CPI), or serum lactate. After $24 \mathrm{~h}$, no significant differences were seen in urine output, vasopressor requirement, or mechanical ventilation duration. Hemolysis and transfusion were significantly higher in patients with the Impella LP 2.5 . Overall 30 -day mortality was $46 \%$ in both groups.

Ouweneel et al., in 2017, reported the results from the IMPRESS trial, which randomized 48 patients with cardiogenic shock post-acute myocardial infarction to hemodynamic support with the Impella $\mathrm{CP}$ or an IABP [42]. Device placement occurred either prior to PCI, during PCI, or immediately after PCI. Notably, 92\% of the study population had a history of recent cardiac arrest requiring resuscitation. At 30 days, mortality was similar (50\% vs. $46 \%$ for patients receiving support with the Impella CP or IABP, respectively; $p=0.92$ ). More bleeding events occurred in patients receiving support with the Impella CP. In a recent meta-analysis including 148 patients, Tandemheart or Impella use was not associated with increased survival in patients with cardiogenic shock [43].

\section{Right ventricular support}

Acute RV failure may occur in multiple settings, including acute myocardial infarction, myocarditis, acute decompensated heart failure, acute pulmonary embolism, pulmonary hypertension, post-cardiotomy, post-transplantation, and following LVAD implantation. Devices currently available for RV support are CentriMag (St Jude Medical, Waltham, MA), a centrifugal pump with a magnetically levitated propeller, Impella RP, an axial catheter-based pump, and the PROTEK Duo (Cardiac Assist Inc., Pittsburgh, PA) catheter with an extracorporeal centrifugal pump [38].

The RECOVER RIGHT study evaluated the safety and efficacy of the Impella RP $(4.0 \mathrm{~L} / \mathrm{min}$ of cardiac support) in 30 patients with RV failure refractory to medical therapy [44]. The cohort was divided into patients with RV failure following LVAD implantation and patients with RV failure following acute myocardial infarction or cardiotomy. The primary endpoint of survival to 30 days or hospital discharge was achieved in $73.3 \%$ of the overall study population, with $83.3 \%$ of patients with RV failure following LVAD implantation and $58.3 \%$ of patients with RV failure following cardiogenic shock post-acute myocardial infarction or cardiotomy alive at 30 days or discharge. All discharged patients were alive at 180 days. These results are particularly compelling when considering that prior studies of RVAD devices in patients with cardiogenic shock reported survival rates of $42-57 \%$ at discharge. In 2015, the Impella RP received approval from the FDA for adult and pediatric patients with a body surface area (BSA) $\geq 1.5 \mathrm{~m}^{2}$ with a diagnosis of acute RV failure following LVAD implantation, acute myocardial infarction, heart transplantation or cardiotomy [38].

The CentriMag (Levitronix LLC, Waltham, MA, USA) is a short- to intermediate support device composed of a centrifugal pump, an electric motor, and a console. It can generate up to $9.9 \mathrm{~L} / \mathrm{min}$ continuous flow at 5000 $\mathrm{rpm}$. The cannulae are inserted through a midline sternotomy, with the inflow cannula in the left ventricle or right superior pulmonary vein and the outflow cannula in the aorta. It is a therapeutic choice for bridging patients with acute cardiogenic shock to longer term mechanical support or transplantation or when inotropic and IABP support fail [45].

\section{VA-ECMO}

VA-ECMO is a form of heart-lung bypass machine that offers extended support to patients whose heart and/or lungs are unable to sustain life in the acute setting. VA-ECMO provides cardiopulmonary support for patients in refractory cardiogenic shock as a bridge to myocardial recovery, bridge to decision, bridge to durable mechanical circulatory support, bridge to heart transplant or bridge to decision for palliative therapy. Important advances in pump and oxygenator technology, percutaneous cannulation techniques and critical care management have enabled ECMO to be considered as a viable lifesaving modality [46].

Over 87,000 patients have been enrolled in the Extracorporeal Life Support Organization (ELSO) registry, including 12,566 adults with VA-ECMO, with the number of VA-ECMO centers increasing considerably in the last decade [46]. In a VA-ECMO circuit, deoxygenated blood is pulled from the venous circulation by a pump via a large cannula. Patients may be cannulated centrally (open chest) or peripherally. Blood passes through the pump into an oxygenator where gas exchange occurs (carbon dioxide removal and oxygenation). Oxygenated blood returns via another cannula to the arterial circulation using a centrifugal pump. Modern centrifugal pumps cause less blood damage, reduced heat generation, cause less thrombogenicity and are smaller than old roller pumps. Polymethyl pentene-coated nonporous hollow fiber membrane oxygenators require lower priming volumes and have better gas exchange capability, 
improved blood compatibility, and show greater stability and preservation of coagulation factors and platelets.

Modern percutaneous approaches have resulted in wider utilization of ECMO, including in-hospital based programs that place patients in cardiac arrest on ECMO support (extracorporeal cardiopulmonary resuscitation [eCPR]), delivery programs with 'in the field' ECMO cannulation, and periprocedural ECMO in cardiac catheterization laboratories, in the surgical room and in the ICU [47]. Distal perfusion catheters that direct a proportion of the returned oxygenated blood flow from the ECMO circuit to the cannulated leg decrease the risks of critical limb ischemia in femoral cannulation.

Survival among patients on VA-ECMO support remains modest, with hospital mortality rates of $50-60 \%$ and 6-month survival as low as $30 \%$ [38]. This may be a consequence of inadequate selection of potential candidates and insufficient training of the involved professionals.

The appropriate selection of patients for ECMO is challenging, because it should consider the adequate support, patient characteristics and the time of the procedure regarding potential reversibility of the shock state. There are few clinical studies evaluating VA-ECMO in adults. Of 12 studies, 7 are retrospective, 2 are meta-analyses and 3 are prospective studies [46]. Patients with potentially reversible causes of shock, such as fulminant myocarditis or primary graft failure, have better survival than patients with cardiogenic shock after surgery or acute myocardial infarction $[48,49]$. Patients for whom ECMO is deployed during or immediately after cardiac arrest have an especially poor prognosis. Pre-ECMO risk factors independently associated with poor outcomes include older age, female sex, and higher body mass index, as well as markers of illness severity including renal, hepatic, or central nervous system dysfunction, longer duration of mechanical ventilation, elevated lactate levels, and reduced prothrombin activity [48].

The management of patients receiving ECMO is based on predefined protocols, which include adequate set and flow control, management of gas exchange, reduction of LV preload, monitoring volume status and anticoagulation and evaluation for weaning [50]. Echocardiography is recommended on a daily basis to evaluate patients receiving VA-ECMO.

\section{Role of a cardiogenic shock team}

Cardiogenic shock is a disease with high mortality and its management is complex, so it is essential to make an early diagnosis and to mobilize adequate resources. The availability of a shock team facilitates timely interventions and avoids futility. The shock team comprises a multidisciplinary group involving an interventional cardiologist, cardiologist, cardiothoracic surgeon, a critical care physician and specialized nurses. The shock team must be ready not only to select and insert the appropriate device in the acute setting, but must also consider long-term strategies, such as permanent devices and transplantation. The shock team must also be prepared to recommend against a mechanical circulatory support device in special settings, such as the occurrence of multiple organ failure, or in the presence of advanced comorbidities.

\section{Conclusion: The future}

Cardiogenic shock should be considered a high-mortality disease that develops as a continuum from the initial insult to the occurrence of organ failure and death. Many advances have contributed to improve diagnosis and therapy of cardiogenic shock. However, these have not led to improved outcomes. In the future, to obtain better results, we should learn how to deliver existing effective therapies within a timely and multifaceted strategy.

Early utilization of mechanical circulatory support instead of escalating doses of inotropes and vasopressors might avoid the downward spiral seen in patients with cardiogenic shock and resulting in high mortality rates. Appropriate device selection is still a complex decision process and we expect to obtain more objective data in the near future to help in the decision process, taking into account simultaneously the severity of cardiogenic shock, goals of care, patient-specific risks and technical limitations along with assessment for futility of care.

\section{Acknowledgements \\ The authors would like to acknowledge Mrs. Stephanie Itala Rizk \\ (Universidade de São Paulo) for her assistance in preparing the images used \\ in this review. \\ Funding \\ Publications costs were funded by Instituto do Coracao.}

Availability of data and materials

Not applicable.

Authors' contributions

The authors wrote, revised and approved the final manuscript.

Ethics approval and consent to participate

Not applicable.

Consent for publication

Not applicable.

Competing interests

The authors declare that they have no competing interests.

\section{Publisher's Note}

Springer Nature remains neutral with regard to jurisdictional claims in published maps and institutional affiliations.

\section{Author details}

'Department of Cardiopneumology, Instituto do Coracao, Universidade de São Paulo, Hospital SirioLibanes, São Paulo, Brazil. ${ }^{2}$ Service de Reánimation 
Médicale, Hôpitaux Universitaires Paris-Sud, Hôpital de Bicêtre, Universite Paris-Sud, Paris, France.

\section{Published online: 09 March 2019}

\section{References}

1. Mebazaa A, Combes A, Van Diepen S, et al. Management of cardiogenic shock complicating myocardial infarction. Intensive Care Med. 2018;44:760-73.

2. Ibanez B, James S, Agewall S, et al. 2017 ESC guidelines for the management of acute myocardial infarction in patients presenting with ST-segment elevation: the Task Force for the management of acute myocardial infarction in patients presenting with ST-segment elevation of the European Society of Cardiology (ESC). Eur Heart J. 2018;39:119-77.

3. van Diepen S, Katz JN, Albert NM, et al. Contemporary management of cardiogenic shock: a scientific statement from the American Heart Association. Circulation. 2017;136:e232-68.

4. Mandawat A, Rao SV. Percutaneous mechanical circulatory support devices in cardiogenic shock. Circ Cardiovasc Interv. 2017;e004337:10.

5. Bellumkonda L, Gul B, Masri SC. Evolving concepts in diagnosis and management of cardiogenic shock. Am J Cardiol. 2018;122:1104-10.

6. Hochman JS, Buller CE, Sleeper LA, et al. Cardiogenic shock complicating acute myocardial infarction —etiologies, management and outcome: a report from the SHOCK trial registry. SHould we emergently revascularize occluded coronaries for cardiogenic shock? J Am Coll Cardiol. 2000;36(3 Suppl A):1063-70.

7. Reynolds HR, Hochman JS. Cardiogenic shock: current concepts and improving outcomes. Circulation. 2008;117:686-97.

8. Harjola VP, Lassus J, Sionis A, et al. Clinical picture and risk prediction of short-term mortality in cardiogenic shock. Eur J Heart Fail. 2015;17:501-9.

9. Ince C. Hemodynamic coherence and the rationale for monitoring the microcirculation. Crit Care. 2015;19:S8.

10. Edul VS, Enrico C, Laviolle B, et al. Quantitative assessment of the microcirculation in healthy volunteers and in patients with septic shock. Crit Care Med. 2012;40:1443-8.

11. Trzeciak S, McCoy JV, Phillip Dellinger R, et al. Early increases in microcirculatory perfusion during protocol-directed resuscitation are associated with reduced multi-organ failure at $24 \mathrm{~h}$ in patients with sepsis. Intensive Care Med. 2008:34:2210-7.

12. Jozwiak M, Monnet $X$, Teboul JL. Less or more hemodynamic monitoring in critically ill patients. Curr Opin Crit Care. 2018;24:309-15.

13. Price $S$, Platz E, Cullen $L$, et al. Expert consensus document: echocardiography and lung ultrasonography for the assessment and management of acute heart failure. Nat Rev Cardiol. 2017;14:427-40.

14. De Backer D, Biston P, Devriendt J, et al. Comparison of dopamine and norepinephrine in the treatment of shock. N Engl J Med. 2010;362:779-89.

15. Hamzaoui $\mathrm{O}$, Jozwiak M, Geffriaud T, et al. Norepinephrine exerts an inotropic effect during the early phase of human septic shock. Br J Anesth 2018;120:517-24.

16. Mclntyre WF, Um KJ, Alhazzani W, et al. Association of vasopressin plus catecholamine vasopressors vs catecholamines alone with atrial fibrillation in patients with distributive shock: a systematic review and meta-analysis. JAMA. 2018;319:1889-900.

17. Levy B, Perez P, Perny J, et al. Comparison of norepinephrine-dobutamine to epinephrine for hemodynamics, lactate metabolism, and organ function variables in cardiogenic shock. A prospective, randomized pilot study. Crit Care Med. 2011;450-455(48):39.

18. Tarvasmaki T, Lassus J, Varpula M, et al. Current real-life use of vasopressors and inotropes in cardiogenic shock-adrenaline use is associated with excess organ injury and mortality. Crit Care. 2016;20:208.

19. Levy BC, Clere-Jehl R, Legras A, et al. Epinephrine versus norepinephrine in cardiogenic shock after acute myocardial infarction. J Am Coll Cardiol. 2018; 72:173-82.

20. Léopold V, Gayat E, Pirracchio R, et al. Epinephrine and short-term survival in cardiogenic shock: an individual data meta-analysis of 2583 patients. Intensive Care Med. 2018:44:847-56

21. Toscani $L$, Aya HD, Antonakaki D, et al. What is the impact of the fluid challenge technique on diagnosis of fluid responsiveness? A systematic review and meta-analysis. Crit Care. 2017;21:207.

22. Nakamura RE, Vincent JL, Fukushima JT, et al. A liberal strategy of red blood cell transfusion reduces cardiogenic shock in elderly patients undergoing cardiac surgery. J Thorac Cardiovasc Surg. 2015;150:1314-20.
23. Hajjar LA, Fukushima JT, Almeida JP, et al. Strategies to reduce blood transfusion: a Latin-American perspective. Curr Opin Anaesthesiol. 2015;28:81-8.

24. Cecconi M, De Backer D, Antonelli M, et al. Consensus on circulatory shock and hemodynamic monitoring.Task force of the European Society of Intensive Care Medicine. Intensive Care Med. 2014;40:1795-815.

25. Marik PE. Obituary: pulmonary artery catheter 1970 to 2013. Ann Intensive Care. 2013;3:38.

26. De Backer D, Hajjar LA. Pinsky MR. is there still a place for the swan-Ganz catheter? We are not sure. Intensive Care Med. 2018;44:960-2.

27. De Backer D, Bakker J, Cecconi M, et al. Alternatives to the swan-Ganz catheter. Intensive Care Med. 2018;44:730-41.

28. Hochman JS, Sleeper LA, Webb JG, et al. Early revascularization in acute myocardial infarction complicated by cardiogenic shock. SHOCK investigators. Should we emergently revascularize occluded coronaries for cardiogenic shock. N Engl J Med. 1999;341:625-34.

29. Hochman JS, Sleeper LA, Webb JG, et al. Early revascularization and longterm survival in cardiogenic shock complicating acute myocardial infarction. JAMA. 2006;295:2511-5.

30. Thiele $\mathrm{H}$, Akin I, Sandri M, et al. PCl strategies in patients with acute myocardial infarction and cardiogenic shock. N Engl J Med. 2017;377:2419-32.

31. de Waha S, Jobs A, Pöss J, et al. Multivessel versus culprit lesion only percutaneous coronary intervention in cardiogenic shock complicating acute myocardial infarction: a systematic review and meta-analysis. Eur Heart J. 2018;7:28-37.

32. Thiele $\mathrm{H}$, Akin I, Sandri M, et al. One-year outcomes after $\mathrm{PCl}$ strategies in cardiogenic shock. N Engl J Med. 2018;379:1699-710.

33. Kapelios CJ, Terrovitis JV, Nanas JN. Current and future applications of the intra-aortic balloon pump. Curr Opin Cardiol. 2014;29:258-65.

34. Thiele H, Zeymer U, Neumann FJ. Intraaortic balloon support for myocardial infarction with cardiogenic shock. N Engl J Med. 2012;367:1287-96.

35. Ibanez B, James S, Agewall S, et al. 2017 ESC Guidelines for the management of acute myocardial infarction in patients presenting with ST-segment elevation. Eur Heart J. 2017;9:119-177.

36. Romeo F, Acconcia MC, Sergi D, et al. The outcome of intra-aortic balloon pump support in acute myocardial infarction complicated by cardiogenic shock according to the type of revascularization: a comprehensive metaanalysis. Am Heart J. 2013;165:679-92.

37. Aso S, Matsui $H$, Fushimi $K$, et al. The effect of intraaortic balloon pumping under venoarterial extracorporeal membrane oxygenation on mortality of cardiogenic patients: an analysis using a nationwide inpatient database. Crit Care Med. 2016;44:1974-9.

38. Mandawat A, Rao SV. Percutaneous mechanical circulatory support devices in cardiogenic shock. Circ Cardiovasc Interv. 2017;e004337:10.

39. Thiele H, Sick P, Boudriot E, et al. Randomized comparison of intra-aortic balloon support with a percutaneous left ventricular assist device in patients with revascularized acute myocardial infarction complicated by cardiogenic shock. Eur Heart J. 2005;26:1276-83.

40. Kar B, Gregoric ID, Basra SS, et al. The percutaneous ventricular assist device in severe refractory cardiogenic shock. J Am Coll Cardiol. 2011;57:688-96.

41. Seyfarth $M$, Sibbing $D$, Bauer I, et al. A randomized clinical trial to evaluate the safety and efficacy of a percutaneous left ventricular assist device versus intra-aortic balloon pumping for treatment of cardiogenic shock caused by myocardial infarction. J Am Coll Cardiol. 2008;52:1584-8.

42. Ouweneel DM, Eriksen E, Sjauw KD, et al. Percutaneous mechanical circulatory support versus intra-aortic balloon pump in cardiogenic shock after acute myocardial infarction. J Am Coll Cardiol. 2017;69:278-87.

43. Thiele $\mathrm{H}$, Jobs A, Ouweneel DM, et al. Percutaneous short-term active mechanical support devices in cardiogenic shock: a systematic review and collaborative meta-analysis of randomized trials. Eur Heart J. 2017:38:3523-31.

44. Anderson MB, Goldstein J, Milano C, et al. Benefits of a novel percutaneous ventricular assist device for right heart failure: the prospective RECOVER RIGHT study of the Impella RP device. J Heart Lung Transplant. 2015;34: 1549-60.

45. Hsu PL, Parker J, Egger C. Mechanical circulatory support for right heart failure: current technology and future outlook. Artif Organs. 2012;36: 332-47.

46. Kleeber ME, Haddad EV, Choi CW, et al. Venoarterial extracorporeal membrane oxygenation in cardiogenic shock. JACC Heart Fail. 2018;6:503-16.

47. Maxhera B, Albert A, Ansari E, et al. Survival predictors in ventricular assist device patients with prior extracorporeal life support: selecting appropriate candidates. Artif Organs. 2014;38:727-32. 
48. Ouweneel DM, Schotborogh JV, Limpens J, et al. Extracorporeal life support during cardiac arrest and cardiogenic shock: a systematic review and metaanalysis. Intensive Care Med. 2016;42:1922-34.

49. Marasco SF, Lukas G, McDonald M, et al. Review of ECMO (extra corporeal membrane oxygenation) support in critically ill adult patients. Heart Lung Circ. 2008;17(Suppl 4):S41-7.

50. Extracorporeal Life Support Organization (ELSO). Guidelines for adult cardiac failure. https://www.elso.org/Portals/0//GD/Archive/FileManager/ e76ef78eabcusersshyerdocumentselsoguidelinesforadultcardiacfailure1.3.pdf. Accessed 31 Oct 2018 